\title{
Distorsiones cognitivas y búsqueda de sensaciones en adultos con ludopatía
}

\author{
Cognitive Distortions and Sensation Seeking in Adults with \\ Compulsive Gambling
}

\author{
Roberto Oropeza-Tena ${ }^{\mathrm{a}}$, María Luisa Ávalos-Latorre ${ }^{\mathrm{b}}$, Alfredo Herrera-Díaz ${ }^{\mathrm{a}}$ \& Sergio Varela-Ramírez ${ }^{\mathrm{a}}$ \\ ${ }^{a}$ Universidad Michoacana de San Nicolás de Hidalgo, Michoacán, México \\ ${ }^{b}$ Universidad de Guadalajara, Guadalajara, México
}

\begin{abstract}
Resumen: Para algunas personas apostar es fundamental en sus vidas y, cuando se hace compulsivamente, puede deteriorar sus relaciones interpersonales, sus actividades cotidianas y su economía. El objetivo de este estudio fue examinar si existen diferencias entre tres grupos de participantes con conductas de juego distintas (peleas de gallos, cartas y máquinas tragamonedas) en los niveles de tres variables dependientes: Gravedad de la ludopatía, Distorsiones cognitivas y Búsqueda de sensaciones. En esta investigación participaron 83 adultos (hombres y mujeres), aficionados a las apuestas. Respondieron instrumentos referentes al juego patológico, distorsiones cognitivas y búsqueda de sensaciones. Al contrario de lo que se esperaba, no hubo diferencias significativas entre los tres grupos de jugadores, ni en los niveles de ludopatía, ni en los niveles de distorsiones cognitivas. No obstante, de acuerdo a las hipótesis, encontramos que el grupo de máquinas tragamonedas presentó puntuaciones significativamente mayores que el grupo de peleas de gallos en la dimensión de desinhibición de la búsqueda de sensaciones.
\end{abstract}

Palabras clave: distorsiones cognitivas, apuestas, búsqueda de sensaciones, ludopatía.
Abstract: For some people, gambling is the most important thing in their lives, and when done compulsively, it may damage their interpersonal relations, daily activities, and financial situation. The aim of this study was to examine whether differences exist between three groups of participants with different gambling behaviors (cockfighting, cards, and slot machines) at the level of three dependent variables: Severity of compulsive gambling, Cognitive distortions, and Sensation seeking. Eighty-three adults (men and women) who like to gamble participated in this study. They answered instruments relating to pathological gambling, cognitive distortions and sensation seeking. Contrary to what expected, there were no significant differences between the three groups of gamblers, nor at the level of gambling or at the level of cognitive distortions. Nevertheless, according to the hypothesis, we found that the slot machine group presented significantly higher scores than the cockfighting group in the dimension of disinhibition in sensation seeking.

Keywords: cognitive distortions, bet, sensation seeking, gambling.

Contacto: M. L. Avalos-Latorre. Centro Universitario de Tonalá, Universidad de Guadalajara. Av. Nuevo Periférico Oriente \#555, Ejido San José Tatepozco, C. P. 45425 Tonalá, Jalisco. Correo electrónico: marilupsi@ hotmail.com

Cómo citar: Oropeza-Tena, R., Ávalos-Latorre, M. L., Herrera-Díaz, A., \& Varela-Ramírez, S. (2016). Distorsiones cognitivas y búsqueda de sensaciones en adultos con ludopatía. Revista de Psicología, 25(1), 1-15. http://dx.doi.org/10.5354/0719-0581.2016.42435 


\section{Introducción}

El juego es un aspecto constitutivo de la vida y del desarrollo del ser humano, sirve como un medio de aprendizaje y ayuda a la interacción con el entorno. En algunos casos, el juego incorpora elementos que lo hacen más atractivo, como el azar y la posibilidad de apostar (Domínguez Álvarez, Pérez Pérez, Sicilia Salcedo, Villahoz González, \& Fernández Alepuz, 2007). Debido a diversos factores biopsicosociales, los jugadores pueden llegar a desarrollar caracteres patológicos con consecuencias graves para ellos mismos y el entorno, obstaculizando significativamente el desarrollo de una vida normal (Villoria López, 2003).

Moliner Castellano (2015) señala que el juego patológico se reconoció como un problema psicológico o mental por primera vez en 1980, siendo la adicción de mayor frecuencia en los años noventa y estando ubicado entre los trastornos del control de impulsos.

Sproston, Erens y Orford (2000) encontraron que en América Latina, entre 5 y 20 millones de personas eran adictas al juego; es decir, entre $1,37 \%$ y $3,37 \%$ de la población. Asimismo, refirieron que Estados Unidos era el país que presentaba un mayor porcentaje de jugadores patológicos, con 3 millones de personas, a la par que Argentina, siguiéndole México, con 2 millones de jugadores patológicos, mientras que en el Reino Unido, las cifras llegaban a 370 mil casos, con posibilidad de aumentar a 700 mil en los años siguientes. Consideraron que en Canadá y Australia la población era propensa a adquirir esta problemática, con un $2 \%$ y el $4 \%$ respectivamente de la población adulta (360 mil a 720 mil personas). Por su parte, y siendo el dato más recientemente reportado, Cía (2013) afirmó que la prevalencia mundial del juego patológico va de $0,5 \%$ a 1,5\% de la población.

En la quinta versión del Manual Diagnóstico y Estadístico de los Trastornos Mentales (Diagnostic and Statistical Manual of Mental Disorders; American Psychiatric Association, 2013) se ha propuesto una nueva categoría denominada trastornos adictivos y relacionados a sustancias. Dentro de este capítulo, se incluyen los trastornos relacionados y no relacionados al consumo de drogas; esta última categoría está compuesta por el juego patológico o ludopatía. En este sentido, Cía (2013) afirma que actualmente las adicciones no se limitan a las conductas generadas por el consumo de sustancias, sino que existen hábitos de conducta aparentemente inofensivos que, en determinadas circunstancias, pueden convertirse en adicciones e interferir gravemente en la vida cotidiana de las personas afectadas, tal es el caso del juego patológico.

Se ha encontrado que los individuos diagnosticados con juego patológico, tienen algunas de las siguientes características: son varones, con bajo nivel de estudios, casados, con trabajo fijo, ingresos propios, consumo de sustancias (alcohol, tabaco o drogas ilegales), impulsividad, trastornos mentales (depresión, ansiedad, trastorno obsesivo-compulsivo), antecedentes de familiares con juego patológico, violencia con la pareja y búsqueda de placer (Afifi, Nicholson, Martins, \& Sareen, 2016; Cano Bedoya \& Pérez Pérez, 2010; Castaño et al., 2011; Dowling et al., 2016; Ferrández de la Cruz, 2008; Moliner Castellano, 2015; Ruiz Pérez, 2014).

Domínguez Álvarez (2009) estableció que una de las características principales del jugador patológico es la presencia frecuente de pensamientos distorsionados o 
irracionales. Beck (1967) denominó distorsiones cognitivas a las interpretaciones incongruentes y exageradas de la realidad. Ante situaciones complejas, como las apuestas, es muy común la tendencia a sesgar la información para simplificarla, pudiendo llegar a pensamientos erróneos (Mañoso, Labrador, \& Fernández-Alba, 2004).

Sánchez Hervás (2003) afirma que cuanto mayor sea la implicación de una persona en un determinado juego, se producirá mayor cantidad de pensamientos irracionales en relación con este. Además, hay una tendencia a sobrevalorar las ganancias, mientras que las pérdidas son minimizadas. Mañoso et al. (2004) han confirmado la presencia de estas distorsiones en diversas investigaciones realizadas con ludópatas, utilizando básicamente dos procedimientos: el primero consiste en analizar las verbalizaciones de los jugadores mientras están jugando; mientras que el segundo, consiste en desarrollar autoinformes retrospectivos.

Además de la presencia de distorsiones cognitivas, las personas con ludopatía también buscan emociones intensas, es decir, tienden a mostrar interés por las situaciones de riesgo que, a la vez, les generan una gran cantidad de estimulación. La búsqueda de sensaciones es un rasgo de personalidad que, de acuerdo a Zuckerman (1979), constituye una necesidad de experimentar sensaciones variadas y complejas, así como el deseo de correr riesgos físicos y sociales, por el simple hecho de disfrutar de tales experiencias.

Tal como sucede con la investigación en torno a las distorsiones cognitivas en los juegos de apuestas, son pocos los estudios realizados sobre búsqueda de sensaciones. Horvath y Zuckerman (1996) evaluaron, en 447 estudiantes universitarios, la relación entre la búsqueda de sensaciones, la conducta de riesgo y la impulsividad (ambos rasgos de los jugadores compulsivos). Sus resultados mostraron que la conducta percibida de los compañeros y la búsqueda de sensaciones, eran fuertes predictoras de la conducta de riesgo, particularmente en las áreas de conducta criminal y violación social.

Más recientemente, Ruíz-Pérez (2009) llevó a cabo un estudio sobre la prevalencia de juego patológico y algunas variables asociadas, en una muestra no aleatoria de usuarios de juegos de apuestas. Encontró que un $49,1 \%$ de los sujetos presentaban algún grado de juego patológico, así también, observó que a mayor grado de juego patológico, mayor variedad en juegos, mayor consumo de alcohol en fines de semana y más demanda de dinero.

Latorre Román, Cámara Pérez y García Pinillos (2014) analizaron la relación existente entre la búsqueda de sensaciones y el consumo de alcohol, tabaco y práctica deportiva en adolescentes de secundaria. Sus hallazgos indicaron que la búsqueda de excitación y desinhibición son factores moderadamente predictivos del consumo de tabaco y alcohol. Un cuarto estudio, realizado en el año 2015 por Alcázar, Verdejo, Bouso y Ortega, analizó la relación entre la búsqueda de sensaciones, riesgo y recompensa, y sus vinculaciones con las conductas externalizadoras (antisocial, de riesgo y de consumo de drogas). Concluyeron que la maduración en los adolescentes del sistema dopaminérgico de recompensa, podría vincularse con rasgos como la impulsividad y la búsqueda de sensaciones.

Tal como puede observarse, son escasos los estudios en torno al juego patológico y 
sus variables psicológicas relacionadas; se observan hallazgos ambiguos y aislados en distintas partes del mundo, además de estar principalmente centrados en el juego de máquinas tragamonedas, dejando de lado otros tipos de juegos que son practicados con frecuencia, como las cartas y las peleas de gallos. No se identificaron investigaciones en las que se aborden el trastorno por juego de apuestas en distintos juegos de azar y su identificación, tipificación y relación de distorsiones cognitivas y búsqueda de sensaciones.

El objetivo de este estudio fue examinar si existen diferencias entre tres grupos de participantes -hombres y mujeres- con conductas de juego distintas: las peleas de gallos, cartas y máquinas tragamonedas; en los niveles de tres variables dependientes: Gravedad de la ludopatía, Distorsiones cognitivas y Búsqueda de sensaciones. La hipótesis es que hay diferencias significativas entre los tres grupos de participantes y entre hombres y mujeres en las tres variables dependientes.

\section{Método}

\section{Participantes}

Colaboraron 83 personas adultas, 59\% hombres y $41 \%$ mujeres. Los criterios de inclusión fueron los siguientes: que fueran personas mayores de 18 años, que participaran al menos tres veces a la semana en alguno de los siguientes juegos: peleas de gallos $(M=34$ años de edad, $D E=4,0)$, cartas $(M=31$ años de edad, $D E=3,0)$ o máquinas tragamonedas $(M$ $=32$ años de edad, $D E=4,0) \mathrm{y}$, finalmente, que no estuviesen realizando algún tratamiento psicológico o psiquiátrico de cualquier tipo.

Los criterios de exclusión fueron: estar bajo los efectos del alcohol o alguna otra sustancia, reportar tener algún problema psicológico y/o estar diagnosticado clínicamente. Por otra parte, se excluyeron aquellos participantes que dejaron inconclusos los cuestionarios y que declinaron participar en algún momento durante la recolección de la información.

El muestreo fue no probabilístico por conveniencia. Los participantes se distribuyeron en tres grupos, de acuerdo al tipo de juego en el participaban con mayor frecuencia y que reportaron era su preferido. Al grupo de peleas de gallos se asignaron 21 participantes (19 hombres y 2 mujeres), 34 participantes al grupo de juego de cartas (22 hombres y 12 mujeres) y 28 al grupo de máquinas tragamonedas (2 hombres y 20 mujeres).

\section{Instrumentos}

Se utilizó el Cuestionario de Juego Patológico de South Oaks (SOGS), versión española (Echeburúa Odriozola et al., 1994), instrumento autoaplicable, cuyo objetivo es evaluar de manera estructurada y cuantificable las características que indican la presencia de juego patológico. Cuenta con un total de 20 reactivos y la persona debe marcar la respuesta que considere que refleja mejor su modo de actuar.

El contenido de cada reactivo se relaciona con las conductas de juego, las fuentes de obtención de dinero para jugar o pagar deudas, y las emociones implicadas. Dicho instrumento discrimina adecuadamente entre población sana y jugadores patológicos, con un alfa de Cronbach de ,98. Respecto a la interpretación, hay tres puntos de corte: sin problemas con el juego, con problemas moderados y jugadores patológicos.

Así mismo, se utilizó el Inventario de Pensamiento sobre Juego (Echeburúa 
Odriozola \& Báez Gallo, 1994), el cual tiene por objetivo evaluar las distorsiones cognitivas de los jugadores patológicos. Incluye 30 reactivos en dos áreas: sesgos cognitivos más característicos y pensamientos racionales sobre esta actividad. Quienes juegan deben valorar en qué medida cada frase es cierta en una escala de cinco puntos (de $1=$ No lo pienso nunca, a 5 = Lo pienso siempre), la escala tiene un alfa de Cronbach de ,82 y está dividida en siete dimensiones:

1. Ilusión de control: creencia en que los resultados del juego dependen más de la propia actividad que del azar.

2. Suerte como responsable de los resultados: la suerte personal se considera un factor predictivo o explicativo de los resultados del juego.

3. Supersticiones: asociaciones accidentales entre un evento o conducta y un premio, de tal forma que el jugador llega a creer que dicho evento aumenta la probabilidad de ganar.

4. Predicciones: augurios particulares sobre los futuros resultados atendiendo a las jugadas anteriores, a una sensación o a una intuición.

5. Azar como proceso autocorrectivo: creencias en que la probabilidad de un evento futuro aumenta cuanto mayor es la racha anterior del suceso contrario.

6. Perder por poco: el jugador cree que "ha estado cerca" del premio y, como consecuencia, que hay más probabilidades de que salga próximamente.

7. Fijación en las frecuencias absolutas: se mide el éxito en el juego, considerando solo cuanto se gana, sin tener en cuenta lo que ha perdido.

El instrumento considera tres subescalas: a) Sesgos cognitivos en relación con la conducta de juego, b) Sesgos cognitivos en relación con la adicción al juego y, c) Información cualitativa adicional. Para obtener la puntuación total en el inventario, se suman las puntuaciones de la subescala a y b; por su parte, la subescala c no contribuye a la puntuación total del inventario.

Finalmente, también se utilizó la Escala Búsqueda de Sensaciones (Scale Sensation Seeking; Zuckerman, Eyseck, \& Eyseck, 1978) que consta de 40 reactivos que el participante debe contestar afirmativa o negativamente (elección forzosa entre dos alternativas). Incluye cuatro subescalas de 10 reactivos cada una:

1. Búsqueda de excitación (BEX): refleja el deseo de encontrar sensaciones y experiencias nuevas, a través de la mente, los sentidos y por estilos de vida inconformistas o poco convencionales.

2. Búsqueda de emociones (BEM): se define por reactivos que expresan el deseo de implicarse en deportes riesgosos o en otras actividades físicamente peligrosas.

3. Desinhibición (DES): refiere a la búsqueda de sensaciones a través de actividades sociales, diversión o sexo.

4. Susceptibilidad hacia el aburrimiento $(\mathrm{SAB})$ : refiere a una escasa tolerancia a las experiencias repetitivas o rutinarias.

No existen puntos de corte propuestos, a mayor puntuación, mayor es la presencia del rasgo. Tiene un alfa de Cronbach de, 90 .

\section{Diseño y procedimiento}

La presente investigación es cuantitativa, descriptiva y transversal de evaluación única. Para la obtención de la muestra, se asistió a establecimientos en donde se llevan a cabo los juegos de 
apuestas (casinos, billares, palenques, entre otros), con el fin de identificar a los participantes potenciales. Con quienes aceptaron participar, se concertó una cita en su domicilio. La recolección de la información se hizo de manera individual a modo de entrevista. Estas se realizaron en un espacio cómodo y aislado de distractores ambientales o de otras personas. Cada entrevista duró aproximadamente 40 minutos. Al concluir el estudio, se hizo una devolución de los resultados obtenidos a cada participante que lo hubiera solicitado. Además, se ofrecieron rutas de intervención para la problemática estudiada.

\section{Consideraciones éticas}

El presente trabajo se ajusta a los artículos 47, 48 y 49 del Código Ético del Psicólogo (Sociedad Mexicana de Psicología, 2009), a los Principios Éticos de Helsinki (Asociación Médica Mundial, 2008) y a los criterios de la Ley General de Salud (Congreso de los Estados Unidos Mexicanos, 2016) en materia de investigación para la salud, en su apartado Título primero de investigación en seres humanos, donde se clasifica este estudio como de riesgo mínimo. Una consideración ética a tomar en cuenta, fue derivar a aquellas personas que se pudieran encontrar en riesgo a las instancias correspondientes para su atención. Todos los sujetos autorizaron su participación y el uso de la información, firmando un consentimiento informado, tanto para hacer investigación como para publicarse en artículos científicos, guardando siempre su anonimato.

\section{Análisis de datos}

Se hizo un análisis descriptivo (por género) respecto a los juegos de preferencia, el perfil del SOGS, las distorsiones cog- nitivas y la búsqueda de sensaciones. Para examinar si existían diferencias entre los tres grupos de participantes en las variables dependientes (Gravedad de la ludopatía, Distorsiones cognitivas y Búsqueda de sensaciones), se llevaron a cabo tres pruebas de Kruskal Wallis (debido a que las variables estaban en nivel ordinal y a que en todos los grupos el $n$ fue menor a 30 ).

\section{Resultados}

\section{Descripción de la muestra}

Tras la realización de los análisis estadísticos, se obtuvieron los datos descriptivos correspondientes a las variables evaluadas. Respecto al juego de preferencia, se observó que el $44,9 \%$ de los hombres prefirió las cartas, el 16,3\% las máquinas tragamonedas y el $39,8 \%$ las peleas de gallos. Por su parte, el 58,8\% de las mujeres prefirió las máquinas tragamonedas, el $35,3 \%$ el juego de cartas y el 5,9\% las peles de gallos.

Los participantes que eligieron el juego de cartas, se iniciaron a edades más tempranas en este $(M=17,0$ años, $D E=4,0$ años), en comparación con los que prefirieron las peleas de gallos $(M=18,0$ años, $D E=4,0$ años) y máquinas tragamonedas $(M=21,0$ años, $D E=10,0$ años $)$. Para el caso de los hombres, el tiempo promedio jugado fue mayor en las peleas de gallos $(M=24,8$ años, $D E=13,1)$, seguido de las cartas $(M=19,4$ años, $D E=8,6)$ y de las máquinas tragamonedas $(M=8,9$ años, $D E=8,0)$; mientras que para las mujeres, fue mayor el tiempo jugando cartas $(M=25,3$ años, $D E=15,6)$, seguido de las máquinas tragamonedas $(M=$ $12,2$ años, $D E=10,2)$ y de las peleas de gallos $(M=10,0$ años, $D E=1,4)$. 
Con respecto al perfil obtenido mediante el SOGS, el $61 \%$ de los participantes presentó problemas moderados de juego, mientras que el $39 \%$ fue considerado jugador/a patológico/a, lo cual, de alguna manera, era de esperarse a partir de los criterios de inclusión considerados en la investigación. Es en las peleas de gallos donde los hombres presentaron el mayor problema (38\% de la muestra), mientras que las mujeres lo presentaron en el juego de cartas $(58,8 \%$ de la muestra $)$.

En cuanto a las Distorsiones cognitivas, se observó que en los tres grupos la mayoría de los participantes presentó un nivel moderado de distorsión, seguido de nivel alto $\mathrm{y}$, por último, de nivel bajo. Tanto hombres como mujeres presentaron el nivel más alto de distorsión cognitiva en las cartas. Aunado a lo anterior, se observó que las dimensiones que ob- tuvieron el nivel más alto fueron: Creencias en la suerte, seguida de Perder por poco y de Azar como proceso autocorrectivo, independiente del tipo de juego preferido (ver tabla 1).

Respecto a la Búsqueda de sensaciones, las mayores puntuaciones se observaron en los varones que prefirieron el juego de cartas, seguidos de las mujeres que prefirieron las máquinas tragamonedas. Respecto de las dimensiones de esta variable, se identificó que una alta proporción de los hombres presentaron puntajes altos en las dimensiones: Desinhibición y Búsqueda de emociones, en el caso de las cartas. Mientras que en las mujeres, se observaron puntajes altos en las dimensiones: Búsqueda de excitación, en las peleas de gallos, y Búsqueda de emociones, en las máquinas tragamonedas (ver tabla 2).

Tabla 1

Distribución porcentual de los participantes de acuerdo al tipo de juego preferido en cada dimensión de distorsión cognitiva

\begin{tabular}{llccccccc}
\hline \multirow{2}{*}{ Juego de apuestas } & Nivel de & \multicolumn{7}{c}{ Porcentajes } \\
& distorsión cognitiva & D1 & D2 & D3 & D4 & D5 & D6 & D7 \\
\hline Juego de cartas $(n=34)$ & Baja & 18 & 15 & 26 & 35 & 27 & 41 & 12 \\
& Moderada & 50 & 56 & 62 & 38 & 27 & 24 & 27 \\
& Alta & 32 & 29 & 12 & 27 & 47 & 35 & 62 \\
Máquina tragamonedas $(n=28)$ & Baja & 29 & 11 & 25 & 18 & 11 & 29 & 4 \\
& Moderada & 57 & 61 & 68 & 61 & 39 & 54 & 57 \\
Pelea de gallos $(n=21)$ & Alta & 14 & 29 & 7 & 21 & 50 & 18 & 39 \\
& Baja & 5 & 14 & 5 & 10 & 10 & 33 & 0 \\
& Moderada & 57 & 76 & 76 & 52 & 24 & 38 & 33 \\
& Alta & 38 & 10 & 19 & 38 & 67 & 29 & 67 \\
\hline
\end{tabular}

Nota: D1 = Ilusión de control; D2 = Predicciones; D3 = Fijación en las frecuencias absolutas; D4 = Azar como proceso autocorrectivo; D5 = Perder por poco; D6 = Supersticiones; D7 = Creencias en la suerte . 
Tabla 2

Distribución porcentual de los participantes de acuerdo al sexo, al juego preferido y el grado de presencia del rasgo de búsqueda de sensaciones.

\begin{tabular}{llcc}
\hline Juego preferido & Grado de presencia & Frecuencia & Porcentaje \\
\hline Hombres & Rasgo alto & 10 & 46 \\
Juego de cartas $(n=22)$ & Rasgo medio & 10 & 46 \\
& Rasgo bajo & 2 & 9 \\
Máquina tragamonedas $(n=8)$ & Rasgo alto & 2 & 25 \\
& Rasgo medio & 3 & 38 \\
Pelea de gallos $(n=19)$ & Rasgo bajo & 3 & 38 \\
& Rasgo alto & 3 & 16 \\
Mujeres & Rasgo medio & 14 & 74 \\
Juego de cartas $(n=12)$ & Rasgo bajo & 2 & 11 \\
& & & \\
& Rasgo alto & 2 & 17 \\
Máquina tragamonedas $(n=20)$ & Rasgo medio & 5 & 42 \\
& Rasgo bajo & 5 & 42 \\
& Rasgo alto & 5 & 25 \\
& Rasgo medio & 7 & 35 \\
Pelea de gallos $(n=2)$ & Rasgo bajo & 8 & 40 \\
& Rasgo alto & 2 & 100 \\
& Rasgo medio & 0 & 0 \\
& Rasgo bajo & 0 & 0 \\
\hline
\end{tabular}

\section{Comparaciones}

De acuerdo a los resultados del análisis de Kruskal Wallis, no resultaron estadísticamente significativas las diferencias entre los tres grupos de participantes y los niveles de juego patológico (ver tabla 3 ). Asimismo, las diferencias entre los tres grupos de jugadores en los niveles de Distorsiones cognitivas tampoco fueron estadísticamente significativas.

Respecto a la existencia de diferencias entre los tres grupos de participantes y las dimensiones de Búsqueda de sensaciones, se observó que, de acuerdo a los resultados del análisis de Kruskal Wallis, solo se presentaron diferencias estadísticamente significativas en la DES y el juego de preferencia (entendido como el juego en el que pasan más tiempo jugando y que fue referido por los participantes), no así en el resto de las dimensiones.

Se compararon los tres grupos de jugadores y la dimensión DES, encontrándose diferencias estadísticamente significativas $(H=6,87, p<, 05)$. En este sentido, para identificar en qué grupos de participantes se encuentran las diferencias en esta dimensión, se realizó un análisis de Mann Whitney, encontrando diferencias entre el grupo de jugadores de máquinas tragamonedas y el de peleas de gallos ( $U$ $=179, p<, 05)$, no así entre los grupos de cartas y máquinas tragamonedas $(U=$ $349,5, p=, 06)$, y de cartas y peleas de gallos $(U=311, p=, 39)$. 
Tabla 3

Resultados del análisis de Kruskal Wallis entre los tres grupos de jugadores en las tres variables.

\begin{tabular}{llcc}
\hline Variable & \multicolumn{1}{c}{ Tipo de jugador } & Rango promedio & $H$ \\
\hline Juego patológico & Cartas & 41,87 & 1,20 \\
& Máquinas tragamonedas & 39,34 & \\
& Peleas de gallos & 45,73 & \\
Distorsiones cognitivas & Cartas & 44,76 & 4,14 \\
& Máquinas tragamonedas & 35,75 & \\
& Peleas de gallos & 45,86 & \\
Búsqueda de sensaciones & & & \\
BEX & Cartas & 37,57 & 4,10 \\
& Máquinas tragamonedas & 41,27 & \\
BEM & Peleas de gallos & 50,14 & \\
& Cartas & 40,53 & 0,25 \\
& Máquinas tragamonedas & 42,98 & \\
DES & Peleas de gallos & 43,07 & \\
& Cartas & 39,63 & $6,87 *$ \\
& Máquinas tragamonedas & 50,63 & \\
SAB & Peleas de gallos & 34,33 & \\
& Cartas & 39,75 & 3,42 \\
& Máquinas tragamonedas & 42,27 & \\
& Peleas de gallos & 37,29 & \\
\hline
\end{tabular}

Nota $:$ BEX = Búsqueda de excitación; $\mathrm{BEM}=$ Búsqueda de emociones; $\mathrm{DES}=$ Desinhibición; $\mathrm{SAB}=$ Susceptibilidad hacia el aburrimiento; $H=$ Estadístico de contraste.

$* p<, 05$.

\section{Discusión y conclusiones}

El objetivo de este estudio fue examinar la existencia de diferencias entre tres grupos de participantes con conductas de juego distintas: peleas de gallos, cartas y máquinas tragamonedas; en los niveles de tres variables dependientes: Gravedad de la ludopatía, Distorsiones cognitivas y Búsqueda de sensaciones.

Al contrario de lo que se esperaba, no hubo diferencias estadísticamente significativas entre los tres grupos de jugadores en los niveles de ludopatía, ni en los niveles de distorsiones cognitivas. No obstan- te, de acuerdo a las hipótesis, se encontró que el grupo de máquinas tragamonedas presentó puntuaciones estadísticamente mayores que el grupo peleas de gallos en la DES.

Dentro de la investigación se encontró que todos los participantes presentaron algún nivel de ludopatía, aunque esto puede deberse a los criterios de inclusión considerados en la investigación. Esto concuerda con los resultados reportados en otras investigaciones, tanto mexicanas como de otros países (Becoña Iglesias, 2004; Becoña Iglesias \& Miguez, 2001; Castaño et al., 2011; Echeburúa \& Fer- 
nández-Montalvo, 2008; Echeburua Odriozola, 1992; Villoria López, 2003), en los cuales se identifica esta problemática psicológica con el mismo instrumento. En este sentido, es claro que el juego patológico es un grave problema de salud pública, sobre todo por estar presente en los distintos niveles socioeconómicos, géneros, grupos culturales, edades u otras variables sociodemográficas.

En la presente investigación se observó que la preferencia por un tipo de juego se relaciona con el género, ya que los hombres tienden a preferir el juego de cartas y las peleas de gallos, mientras que las mujeres se inclinan más por las máquinas tragamonedas. En este sentido, Castaño et al. (2011) y Muñoz-Molina (2008) también reportaron una diferencia significativa entre el tipo de juego y el género. Si bien es cierto que los juegos de apuestas son preferidos por los varones, cada vez es más frecuente que las mujeres se involucren en este tipo de actividades. Aunque existen a la fecha pocos estudios al respecto, se puede observar una tendencia hacia determinados tipos de modalidad de juego en las mujeres (Zuckerman et al., 1978).

Este estudio sugiere que el inicio en los juegos de apuestas es predominantemente en las cartas, lo cual podría deberse a que es una actividad que puede comenzar desde el hogar a edades muy tempranas. En esta línea, en un estudio realizado por Arcaya, Martina, Gutiérrez y Romero (2012), en el cual concluyen que existe una relación inversa entre el nivel de integración familiar y la práctica de juegos de apuestas; esta práctica dentro del núcleo familiar es considerada por los expertos como un factor de riesgo para los integrantes más jóvenes de la familia.

En México una tradición muy arraigada son las peleas de gallos, en el presente estudio fue el juego en el cual se reportó una mayor inversión de tiempo en los hombres, seguido del juego de cartas en las mujeres. Las peleas de gallos van acompañadas de un ambiente muy peculiar, en el que las apuestas son muy altas y, además, están acompañadas frecuentemente por alcohol, otras drogas y prostitución encubierta, elementos que podrían hacer de este juego más atractivo para algunas personas (Arcaya et al., 2012). Además, las peleas de gallos tienen otras peculiaridades, como la duración mayor en comparación con los otros juegos estudiados, la creencia de contar con una estrategia previa, lo sangriento de las peleas y el apego hacia el gallo.

Respecto del nivel de Distorsión cognitiva, aunque fue predominantemente moderado, se aprecia que quienes apuestan en los gallos puntuaron más alto, esto es más notable en los varones que en las mujeres. Los tipos de distorsiones más frecuentes fueron Perder por poco y Creencias en la suerte, este hallazgo fue inesperado, ya que se anticipada que en los participantes que juegan peleas de gallos y cartas, la dimensión Ilusión de control sería la predominante; debido a que son juegos que implican -de cierta manera- determinadas estrategias, lo que contradice los resultados.

Los estudios que se han realizado, en términos de las distorsiones cognitivas en personas con juego patológico, y en los que se ha utilizado el mismo instrumento, son principalmente comparaciones entre personas jugadoras y no jugadoras (Labrador \& Mañoso, 2005; Labrador Encinas, Fernández-Alba Luengo, \& Mañoso Alfaro, 2002; Mañoso et al., 2004), a diferencia del presente estudio, que aborda a participantes con un problema moderado y juego patológico, así como tres juegos de apuestas. 
A pesar de que el tipo de muestra difiere de otras investigaciones, se encontraron algunas similitudes, la más importante es que la dimensión Perder por poco es la distorsión más frecuente en todos los estudios que han abordado el juego patológico, incluyendo el actual. Por otro lado, y en contraposición, se encontró que la dimensión Creencias en la suerte no ha sido reportada como predominante en otras investigaciones, como lo es en este caso.

Existen numerosos estudios en los que se analiza la búsqueda de sensaciones y su relación con el uso de sustancias, principalmente con el alcohol (Echeburúa, Bravo de Medina, \& Aizpiri, 2008; González-Iglesias, Gómez-Fraguela, Gras, \& Planes, 2014; Latorre Román et al., 2014), en estos se ha resaltado que en las personas consumidoras la búsqueda de sensaciones es más alta, en comparación con personas no consumidoras.

En la presente investigación, usando los mismos instrumentos, se encontró algo similar en dicha variable, ya que la mayoría de los participantes tiene la necesidad de descubrir y encontrar experiencias nuevas, variadas y complejas; nuevamente, las personas que prefieren las peleas de gallos fueron las que más altos niveles puntuaron en dicha variable. Esto, probablemente, se deba a las propias características del juego, es común observar en las peleas de gallos aspectos muy interesantes, como el hecho de que es un juego mayoritariamente practicado por el género masculino, donde intervienen otros aspectos psicológicos. Es probable que los altos niveles de distorsión cognitiva favorezcan también que no solo jueguen por dinero y diversión, sino que el jugador, a través del juego, pretenda demostrar "hombría" o "superioridad" con actitudes altaneras y agresivas.
Las sensaciones identificadas con mayor frecuencia fueron la BEX y DES. De acuerdo a Zuckerman, Eysenck y Eysenck (1978), la primera hace referencia al atrevimiento desproporcionado al momento de jugar. Por otro lado, la DES se refiere a la expresión de emociones y actitudes manifestadas durante el juego, reflejando conductas altaneras, egocéntricas y que, en cierto sentido, pretenden hacer alusión a la masculinidad y la búsqueda de la superioridad sobre el rival. La BEX, tal como se ha mencionado a lo largo del escrito, hace referencia a una expresión activa de un rasgo de la personalidad, el cual es el efecto sensorial de la estimulación externa, que cobra mayor importancia para definir su valor como refuerzo primario.

Se puede observar que los jugadores buscan en el juego de cartas, en el caso de los hombres, y en las máquinas tragamonedas, en el caso de las mujeres, aquellos estímulos externos que les permitirían maximizar las sensaciones que les son más placenteras. El alto buscador de sensaciones se caracteriza por su tendencia a hacer cosas que, para los demás, pueden ser peligrosas; de tal manera que la valoración que el jugador patológico hace del riesgo, es mínima ante el juego de apuestas y siempre buscará aquellas sensaciones que le son placenteras e inusuales.

El bajo tamaño de la muestra de todos los grupos que participaron en la investigación, es una limitante para hacer comparaciones y conclusiones más sólidas. También se considera importante continuar el trabajo de adaptación y validación en población mexicana; aunque la calidad de los instrumentos empleados para evaluar las variables psicológicas ha sido alta, debido al trabajo que han hecho investigadores de otros países, y al que so- 
metieron a un escrupuloso proceso de jueceo.

Como líneas de investigación futuras, es recomendable que se indaguen los antecedentes familiares relacionados con la práctica de juegos de apuestas. Por otro lado, es necesario resaltar la carencia de investigaciones científicas sobre las peleas de gallos, lo cual es necesario subsanar, dada su frecuencia y relevancia en países latinoamericanos. Además, los hallazgos en la investigación indican que, en estas actividades, la gravedad del juego patológico y otras alteraciones psicológicas, son importantes y necesitan ser analizadas, no solo aisladamente, sino también en su relación con otras problemáticas, como el consumo de sustancias, la prostitución y rasgos de la personalidad. Este trabajo marca la pauta para otras investigaciones que estudien en profundidad este tipo de problemáticas psicológicas.

\section{Referencias}

Afifi, T. O., Nicholson, R., Martins, S. S., \& Sareen J. (2016). A longitudinal study of the temporal relation between problem gambling and mental and substance use disorders among young adults. Canadian Journal of Psychiatry, 61(2), 102-111. http://dx.doi.org/10.1177/0706743715625950

Alcázar, M. A., Verdejo, A., Bouso, J. C., \& Ortega, J. (2015). Búsqueda de sensaciones y conducta antisocial. Anuario de Psicología Jurídica, 25(1), 75-80. http://dx.doi.org/10.1016/j.apj.2015.01.003

American Psychiatric Association. (2013). Diagnostic and statistical manual of mental disorders (5th ed.). Washington, District of Columbia: American Psychiatric Association.

Arcaya, M., Martina, M., Gutiérrez, C., \& Romero, Y. (2012). Práctica de juegos de azar y su relación con la integración familiar en la población peruana. Revista Peruana de Epidemiología, 16(1), 1-7. Recuperado de https://is.gd/mVQV8p

Asociación Médica Mundial. (2008). Declaración de Helsinki: principios éticos para las investigaciones médicas en seres humanos. Recuperado de http://www.wma.net/es/30publications/10policies/b3/index.html

Beck, A. T. (1967) Depression: Clinical, experimental, and theoretical aspects. New York, New York: Hoeber Medical Division, Harper \& Row.

Becoña Iglesias, E. (2004). El juego patológico: prevalencia en España. Salud y Drogas, 4(2), 9-34. Recuperado de http://www.redalyc.org/pdf/839/83940202.pdf

Becoña Iglesias, E. \& Míguez, M. D. (2001). Juego problema y consumo de drogas ilegales en adolescentes escolarizados. Psicopatología Clínica, Legal y Forense, 1(1), 27-43. Recuperado de http://www.masterforense.com/pdf/2001/2001art2.pdf 
Cano Bedoya, V. H. \& Pérez Pérez, J. A. (2010). La ludopatía y los empleados en la Universidad de San Buenaventura, seccional de Medellín, Colombia - 2010. Agora USB, 10(2), 451-479. Recuperado de

http://dialnet.unirioja.es/servlet/articulo?codigo=3797170

Castaño, S., Castaño Castrillón, J. J., Constanza Cañón, S., Melo, M. A., Mendoza, P. A., Montoya, J. M., ... Velásquez, J. P. (2011). Estudio epidemiológico sobre juegos de azar y factores asociados en población universitaria de la Universidad de Manizales, Colombia, 2010. Archivos de Medicina, 11(2), 101-113. Recuperado de http://www.redalyc.org/pdf/2738/Resumenes/Resumen_273821489003_1.pdf

Cía, A. H. (2013). Las adicciones no relacionadas a sustancias (DSM-5, APA, 2013): un primer paso hacia la inclusión de las adicciones conductuales en las clasificaciones categoriales vigentes. Revista de Neuropsiquiatría, 76(4), 210-217.

http://dx.doi.org/10.20453/rnp.v76i4.1169

Congreso de los Estados Unidos Mexicanos. (2016). Ley General de Salud. Recuperado de http://www.diputados.gob.mx/LeyesBiblio/pdf/142_010616.pdf

Domínguez Álvarez, A. M. (2009). Epidemiología y factores implicados en el juego patológico. Apuntes de Psicología, 27(1), 3-20. Recuperado de http://www.jogoremoto.com/docs/extra/xiR7At.pdf

Domínguez Álvarez, A. M., Pérez Pérez, M. D., Sicilia Salcedo, E., Villahoz González, J., \& Fernández Alepuz, R. (2007). Guía clínica: actuar ante el juego patológico. Andalucía, España: Junta de Andalucía.

Dowling, N., Suomi, A., Jackson, A., Lavis, T., Patford, J., Cockman, S., ... Abbott, M. (2016). Problem gambling and intimate partner violence: A systematic review and metaanalysis. Trauma, Violence, \& Abuse, 17(1), 43-61.

http://dx.doi.org/10.1177/1524838014561269

Echeburúa, E., Bravo de Medina, R., \& Aizpiri, J. (2008). Variables de personalidad, alteraciones psicopatológicas y trastornos de personalidad en pacientes con dependencia de alcohol en función de la tipología de Cloninger. Psicothema, 20(4), 525-530. Recuperado de http://www.psicothema.com/pdf/3518.pdf

Echeburúa, E. \& Fernández-Montalvo, J. (2008). Are there more personality disorders in treatment-seeking pathological gamblers than in other kind of patients? A comparative study between the IPDE and the MCMI. International Journal of Clinical and Health Psychology, 8(1), 53-64. Recuperado de

http://www.redalyc.org/articulo.oa?id=33780104

Echeburua Odriozola, E. (1992). Psicopatología, variables de personalidad y vulnerabilidad psicológica al juego patológico. Psicothema, 4(1), 7-20. Recuperado de http://www.psicothema.com/pdf/812.pdf

Echeburúa Odriozola, E. \& Báez Gallo, C. (1994). Concepto y evaluación del juego patológico. En J. L. Graña Gómez (Ed.), Conductas adictivas: teoría, evaluación y tratamiento (pp. 521-556). Madrid, España: Debate. 
Echeburúa Odriozola, E., Báez Gallo, C., Fernández-Montalvo, J., \& Páez Rovira, D. (1994). Cuestionario de juego patológico de South Oaks (SOGS): validación española. Análisis y Modificación de Conducta, 20(74), 769-791. Recuperado de http://www.ehu.eus/echeburua/pdfs/1-sogs.pdf

Ferrández de la Cruz, A. (2008). Eficacia de un tratamiento psicológico multicomponente de aplicación individual para jugadores patológicos de máquinas recreativas con premio (Tesis doctoral), Universidad Complutense de Madrid, Madrid, España). Recuperado de http://eprints.sim.ucm.es/10166/1/T31527.pdf

González-Iglesias, B., Gómez-Fraguela, J. -A., Gras, M. -E., \& Planes, M. (2014). Búsqueda de sensaciones y consumo de alcohol: el papel mediador de la percepción de riesgos y beneficios. Anales de Psicología, 30(3), 1061-1068.

http://dx.doi.org/10.6018/analesps.30.3.170831

Horvath, P. \& Zuckerman, M. (1996). Búsqueda de sensaciones, valoración y conducta de riesgo. Revista de Toxicomanías, 9, 26-38. Recuperado de http://www.cat-barcelona.com/uploads/rets/RET09_3.pdf

Labrador, F. J. \& Mañoso, V. (2005). Cambio en las distorsiones cognitivas de jugadores patológicos tras el tratamiento: comparación con un grupo control. International Journal of Clinical and Health Psychology, 5(1), 7-22. Recuperado de http://www.aepc.es/ijchp/articulos_pdf/ijchp-130.pdf

Labrador Encinas, F. J., Fernández-Alba Luengo, A., \& Mañoso Alfaro, V. (2002). Relación entre la reducción de las distorsiones cognitivas referidas al azar y la consecución de éxito terapéutico en jugadores patológicos. Psicothema, 14(3), 551-557. Recuperado de http://www.psicothema.com/psicothema.asp?id=764

Latorre Román, P. A., Cámara Pérez, J. C., \& García Pinillos, F. (2014). Búsqueda de sensaciones y hábitos de tabaquismo, consumo de alcohol y práctica deportiva en estudiantes de educación secundaria. Salud Mental, 37(2), 145-152.

http://dx.doi.org/10.17711/sm.0185-3325.2014.018

Mañoso, V., Labrador, F. J., \& Fernández-Alba, A. (2004). Tipo de distorsiones cognitivas durante el juego en jugadores patológicos y no jugadores. Psicothema, 16(4), 576-581. Recuperado de http://www.psicothema.com/psicothema.asp?id=3034

Moliner Castellano, B. (2015). Psicopatología de personalidad y juego patológico (Tesis de licenciatura, Universitat Jaume-I, Castellón de la Plana, España). Recuperado de https://is.gd/b9Ljjm

Muñoz-Molina, Y. (2008). Meta-análisis sobre juego patológico 1997-2007. Revista de Salud Pública, 10(1), 150-159. http://dx.doi.org/10.1590/s0124-00642008000100014

Ruíz-Pérez, J. I. (2009). Juego patológico en usuarios de casinos en Bogotá: prevalencia y relaciones con consumo de alcohol, búsqueda de sensaciones y patrones de juego. Revista Colombiana de Psicología, 18(2), 145-156. Recuperado de http://www.redalyc.org/articulo.oa?id=80412409004 
Ruiz Pérez, J. I. (2014). Juego patológico y dependencia del alcohol en una muestra de trabajadores y estudiantes universitarios: prevalencias, interrelaciones y diferencias de género. Psychologia: Avances de la Disciplina, 8(1), 33-42. Recuperado de http://www.redalyc.org/articulo.oa?id=297231283004

Sánchez Hervás, E. (2003). Juego patológico: un trastorno emergente. Trastornos Adictivos, 5(2), 75-87.

http://dx.doi.org/10.1016/s1575-0973(03)78557-2

Sociedad Mexicana de Psicología. (2009). Código Ético del Psicólogo. Distrito Federal, México: Trillas.

Sproston, K., Erens, B., \& Orford, J. (2000). Gambling behavior in Britain: Results from the British Gambling Prevalence Survey. London, England: The National Centre for Social Research.

Villoria López, C. (2003). Juego patológico en los universitarios de la Comunidad de Madrid. Clínica y Salud, 14(1), 43-65. Recuperado de http://www.redalyc.org/pdf/1806/180617967003.pdf

Zuckerman, M. (1979). Sensation seeking: Beyond the optimal level of arousal. Hillsdale, New Jersey: Lawrence Erlbaum.

http://dx.doi.org/10.1016/0191-8869(80)90044-6

Zuckerman, M., Eysenck, S. B., \& Eysenck, H. J. (1978). Sensation seeking in England and America: Cross-cultural, age, and sex comparisons. Journal of Consulting and Clinical Psychology, 46(1), 139-149.

http://dx.doi.org/10.1037/0022-006x.46.1.139

Fecha de recepción: 1 de diciembre de 2015

Fecha de aceptación: 1 de abril de 2016 